\title{
Mutations of Laurent Polynomials and Flat Families with Toric Fibers ${ }^{\star}$
}

Nathan Owen ILTEN

Department of Mathematics, University of California, Berkeley CA 94720, USA

E-mail: nilten@math.berkeley.edu

URL: http://math. berkeley.edu/ nilten/

Received May 21, 2012, in final form July 25, 2012; Published online July 28, 2012

http://dx.doi.org/10.3842/SIGMA.2012.047

\begin{abstract}
We give a general criterion for two toric varieties to appear as fibers in a flat family over $\mathbb{P}^{1}$. We apply this to show that certain birational transformations mapping a Laurent polynomial to another Laurent polynomial correspond to deformations between the associated toric varieties.
\end{abstract}

Key words: toric varieties; mirror symmetry; deformations; Newton polyhedra

2010 Mathematics Subject Classification: 14M25; 14D06; 53D37

\section{Introduction}

Consider the $n$-dimensional torus $T=\left(\mathbb{C}^{*}\right)^{n}$ with coordinates $z_{1}, \ldots, z_{n}$ along with the logarithmic volume form

$$
\omega=\frac{1}{(2 \pi i)^{n}} \frac{d z_{1}}{z_{1}} \wedge \cdots \wedge \frac{d z_{n}}{z_{n}}
$$

where $z_{i}$ are coordinates on $T$.

Definition 1.1 (cf. [4, Definition 7]). Let $f: T \rightarrow \mathbb{C}$ be a Laurent polynomial in $z_{1}, \ldots, z_{n}$. A mutation of $f$ is a birational transformation $\phi \in \operatorname{Aut}\left(\mathbb{C}\left(z_{1}, \ldots, z_{n}\right)\right)$ preserving $\omega$ such that $\phi(f)$ is again a Laurent polynomial.

Such transformations arise in the context of wall-crossing for counts of holomorphic discs bounded by special Lagrangian tori, see e.g. [2].

Example 1.2. Let $g$ be a non-zero Laurent polynomial in $z_{2}, \ldots, z_{n}$, and consider the birational transformation

$$
\phi:\left(z_{1}, z_{2}, \ldots, z_{n}\right) \mapsto\left(z_{1} / g, z_{2}, \ldots, z_{n}\right) .
$$

Then $\phi$ is a mutation of a Laurent polynomial $f$ if and only if $f$ can be written as

$$
f=\sum_{i=k}^{l} f_{i} z_{1}^{i}
$$

with $f_{i}$ Laurent polynomials in $z_{2}, \ldots, z_{n}$ such that, for $i>0, f_{i} / g^{i}$ is a Laurent polynomial.

\footnotetext{
*This paper is a contribution to the Special Issue "Mirror Symmetry and Related Topics". The full collection is available at http://www.emis.de/journals/SIGMA/mirror_symmetry.html
} 
The purpose of this article is to relate these special mutations to deformations. Given a lattice polytope $\Delta$ containing the origin in its interior, let $\Sigma(\Delta)$ be the face fan of $\Delta$, that is, the fan whose cones are generated by proper faces of $\Delta$. Let $\mathbb{T V}(\Delta)$ denote the toric variety corresponding to the fan $\Sigma(\Delta)$, see [3] for details on toric varieties. Our main result is

Theorem 1.3. Let $\phi$ be a mutation of a Laurent polynomial $f$ of the type of Example 1.2 and suppose that $\Delta(f)$ contains the origin in its interior. Then there is a flat projective family $\pi: \mathcal{X} \rightarrow \mathbb{P}^{1}$ such that $\pi^{-1}(0)=\mathbb{T} \mathbb{V}(\Delta(f))$ and $\pi^{-1}(\infty)=\mathbb{T} \mathbb{V}(\Delta(\phi(f))$.

A special case of the mutations from Example 1.2 are those considered by Galkin and Usnich in [4]. In particular, the above theorem shows that any two toric surfaces related via one of the mutations of [4] appear as special fibers in a flat projective family over $\mathbb{P}^{1}$, answering a question posed in loc. cit.

The family $\pi: \mathcal{X} \rightarrow \mathbb{P}^{1}$ comes from a general construction of $\mathrm{R}$. Vollmert and the author, see [5]. In Section 2, we use this construction to formulate a criterion for two toric varieties to appear as fibers in a flat family over $\mathbb{P}^{1}$, see Theorem 2.1 . We apply this to prove Theorem 1.3 in Section 3. In Section 4 we briefly discuss the mutations of Usnich and Galkin.

We end this section by fixing notation. Let $N$ be a lattice, and $M=\operatorname{Hom}(N, \mathbb{Z})$ its dual. We denote the $\mathbb{Q}$-vector spaces $N \otimes \mathbb{Q}$ and $M \otimes \mathbb{Q}$ by $N_{\mathbb{Q}}$ and $M_{\mathbb{Q}}$, respectively. To any polyhedral cone $\sigma \subset N_{\mathbb{Q}}$, we can associate the affine toric variety $\mathbb{T} \mathbb{V}(\sigma)=\operatorname{Spec} \mathbb{C}\left[\sigma^{\vee} \cap M\right]$. Likewise, for any polytope $\Delta \subset N_{\mathbb{Q}}$ containing the origin in its interior, let $\mathbb{T} \mathbb{V}(\Delta)$ denote the toric variety corresponding to the face fan $\Sigma(\Delta)$ of $\Delta$.

Let $\Delta_{0}, \Delta_{1}$ be polyhedra in $N_{\mathbb{Q}}$. Their Minkowski sum $\Delta_{0}+\Delta_{1}$ consists of all points $v_{0}+v_{1}$ where $v_{0} \in \Delta_{0}, v_{1} \in \Delta_{1}$. Given a polyhedron $\Delta \subset N_{\mathbb{Q}}$, its tailcone tail $(\Delta)$ consists of all $v \in N_{\mathbb{Q}}$ such that $v+\Delta \subset \Delta$.

Definition 1.4. A pair of polyhedra $\Delta_{0}, \Delta_{1}$ in $N_{\mathbb{Q}}$ is admissible if their tailcones are equal, and if for all $u \in M \cap \operatorname{tail}\left(\Delta_{0}\right)^{\vee}$, the minimum value of $u$ on $\Delta_{i}$ is integral for either $i=0$ or $i=1$ (or both).

\section{Families with toric fibers}

We now describe a criterion for two toric varieties $\mathcal{X}_{0}, \mathcal{X}_{\infty}$ to appear as fibers in a flat family $\pi: \mathcal{X} \rightarrow \mathbb{P}^{1}$. For now, we will focus on the case where $\mathcal{X}_{0}$ and $\mathcal{X}_{\infty}$ are affine, as well as making a number of simplifying assumptions.

The idea is to start with $\mathcal{X}_{0}$, and provide a procedure for constructing possible $\mathcal{X}_{\infty}$. So let us fix some affine toric variety $\mathcal{X}_{0}=\mathbb{T} \mathbb{V}(\sigma)$, where $\sigma$ is a cone in $N_{\mathbb{Q}}$. To construct $\mathcal{X}_{\infty}$, we will make three choices, only two of which will have an effect on the end result. First of all, we will choose a primitive $u \in M=\operatorname{Hom}(N, \mathbb{Z})$ such that $\pm u \notin \sigma^{\vee}$. This gives rise to an exact sequence

$0 \longrightarrow N^{\prime} \stackrel{\iota}{\longrightarrow} N \stackrel{u}{\longrightarrow} \mathbb{Z} \longrightarrow 0$.

Note that the choice of $u$ corresponds to a subtorus $T^{\prime}=\mathbb{C}^{*} \otimes N^{\prime}$ of the big torus $T=\mathbb{C}^{*} \otimes N$. Let $s: N \rightarrow N^{\prime}$ be a cosection, that is, $s \circ \iota$ is the identity. Although there is a choice involved here, it will not have any effect on the end result.

From the above exact sequence, we get two polyhedra in $N_{\mathbb{Q}}^{\prime}$ :

$$
\Delta_{0}:=s\left(u^{-1}(1) \cap \sigma\right), \quad \Delta_{\infty}:=s\left(u^{-1}(-1) \cap \sigma\right) .
$$

Note that both $\Delta_{0}$ and $\Delta_{\infty}$ have the tailcone $s((\operatorname{ker} u) \cap \sigma)$.

We can recover $\mathcal{X}_{0}$ from these polyhedra. Let $e$ be a generator of the $\mathbb{Z}$ factor in $N^{\prime} \oplus \mathbb{Z}$. Then up to lattice isomorphism, the cone $\sigma$ is equal to

$$
\operatorname{cone}\left\{\operatorname{tail}\left(\Delta_{0}\right), \Delta_{0}+e, \Delta_{\infty}-e\right\} \subset\left(N^{\prime} \oplus \mathbb{Z}\right)_{\mathbb{Q}} .
$$


We now come to the third choice: let $\Delta_{0}^{0}, \Delta_{0}^{1}$ be an admissible pair of polyhedra such that $\Delta_{0}^{0}+\Delta_{0}^{1}=\Delta_{0}$, and such that the pair $\Delta_{0}^{1}, \Delta_{\infty}$ is also admissible.

Theorem 2.1 (cf. [5, Theorem 2.8]). Under the above assumptions, there is a flat family $\pi: \mathcal{X} \rightarrow \mathbb{P}^{1}$ with $\mathcal{X}_{0}$ the fiber over the origin. The small torus $T^{\prime}$ acts on $\mathcal{X}$, preserving fibers and extending the action on $\mathcal{X}_{0}$. The fiber $\mathcal{X}_{\infty}$ over $\infty$ is toric and isomorphic to $\mathbb{T} \mathbb{V}\left(\sigma_{\infty}\right)$, where

$$
\sigma_{\infty}:=\operatorname{cone}\left\{\operatorname{tail}\left(\Delta_{0}\right), \Delta_{0}^{0}+e, \Delta_{0}^{1}+\Delta_{\infty}-e\right\} \subset\left(N^{\prime} \oplus \mathbb{Z}\right)_{\mathbb{Q}}
$$

Note that up to lattice isomorphism, $\sigma_{\infty}$ doesn't depend on the cosection $s$.

Proof. This is an immediate consequence of the construction of Section 2 in [5], coupled with the observation that the affine base $B$ of the family there may be replaced by $\mathbb{P}^{1}$. More precisely, the polyhedra $\Delta_{0}$ and $\Delta_{\infty}$ form the coefficients of a $p$-divisor $\mathcal{D}$ encoding $\mathcal{X}_{0}$ as a $T^{\prime}$-variety, see Remark 1.8 of loc. cit. Applying [5, Theorem 2.8] to the decomposition $\Delta_{0}=\Delta_{0}^{0}+\Delta_{0}^{1}$ gives a one-parameter flat family over $\mathbb{A}^{1}$ with $\mathcal{X}_{0}$ as the special fiber. Since the pair $\Delta_{0}^{1}, \Delta_{\infty}$ is admissible, this family may in fact be extended to $\mathbb{P}^{1}$, with the fiber over $\infty$ described by the $p$-divisor with coefficients $\Delta_{0}^{0}$ and $\Delta_{0}^{1}+\Delta_{\infty}$. But this is just the toric variety $\mathbb{T} \mathbb{V}\left(\sigma_{\infty}\right)$.

The remark concerning the independence from the cosection $s$ follows from the fact that choosing a different cosection $s^{\prime}$ will shift $\Delta_{0}$ by some lattice element $v \in N^{\prime}$ and $\Delta_{\infty}$ by its opposite $-v$.

Remark 2.2. If $\Delta_{\infty}$ is an integral translate of its tailcone, then the general fiber of $\pi$ is isomorphic to $\mathcal{X}_{\infty}$. However, in general, the general fiber will not be toric, admitting instead only the codimension-one torus action by $T^{\prime}$. The fibers of the family as well as its total space may be described quite explicitly using $p$-divisors, see [1].

There are two ways to generalize the above result to non-affine toric varieties. First of all, instead of considering cones and polyhedra, one may consider fans and polyhedral complexes; see [5, Section 4]. A similar approach may also be found in [6]. We will not discuss this here. Secondly, if we restrict to projective toric varieties, we can put them in flat projective families by considering degree zero deformations of their affine cones. Explicitly, if $\mathcal{X}_{0}=\operatorname{Proj} \mathbb{C}\left[\sigma^{\vee} \cap M\right]$ with $\mathbb{Z}$ grading given by $v \in N$, this means that we must choose $u$ as above such that $u(v)=0$. This is the approach which we will pursue in the remainder of this article.

Example 2.3. Let $N=\mathbb{Z}^{3}$, and consider the cone $\sigma$ generated by $(-1,1,1),(1,1,1)$, and $(0,-1,1)$. The toric variety $\mathbb{T} \mathbb{V}(\sigma)$ is just the affine cone over $\mathbb{P}(1,1,2)$ in its anticanonical embedding, where the grading is given by $v=(0,0,1)$. Now take $u=(0,1,0)$ as in the construction above. Taking the natural cosection coming from the splitting of $\mathbb{Z}^{3}$, the polyhedra $\Delta_{0}, \Delta_{\infty}$ are as pictured in Fig. 1 . There, we also picture polyhedra $\Delta_{0}^{0}, \Delta_{0}^{1}$ which are admissible and sum to $\Delta_{0}$, and such that $\Delta_{0}^{1}, \Delta_{\infty}$ are also admissible. The corresponding cone $\sigma_{\infty}$ is generated by $(-1,1,1),(0,1,1),(0,-1,1)$, and $(1,-1,1)$. The toric variety $\mathbb{T} \mathbb{V}(\sigma)$ is just the affine cone over $\mathbb{P}^{1} \times \mathbb{P}^{1}$ in its anticanonical embedding, where the grading is again given by $v=(0,0,1)$.

Thus we get a flat family $\mathcal{X} \rightarrow \mathbb{P}^{1}$ with $\mathbb{P}(1,1,2)$ and $\mathbb{P}^{1} \times \mathbb{P}^{1}$ as special fibers. Note that since $\Delta_{\infty}$ is a lattice translate of its tailcone, the general fiber of this family is also $\mathbb{P}^{1} \times \mathbb{P}^{1}$. In fact, this is just the classical smoothing of $\mathbb{P}(1,1,2)$ to $\mathbb{P}^{1} \times \mathbb{P}^{1}$.

\section{Proof of main theorem}

In this section, we use Theorem 2.1 to prove Theorem 1.3. Let $g$ be as in Example 1.2 with corresponding birational transformation $\phi$, and suppose that $\phi$ is a mutation of some Laurent 


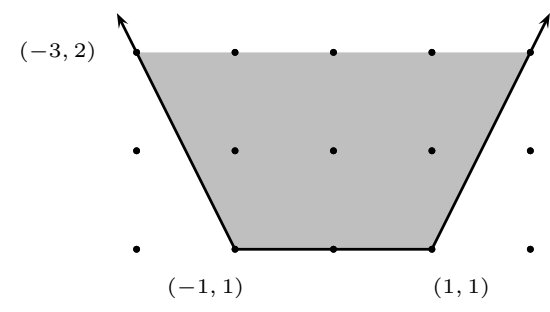

(a) $\Delta_{0}$

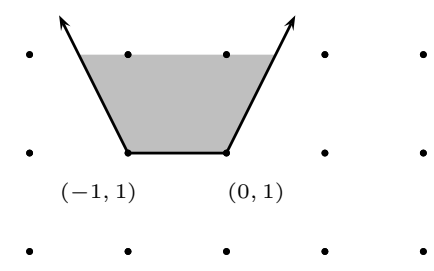

(c) $\Delta_{0}^{0}$
$(3,2)$

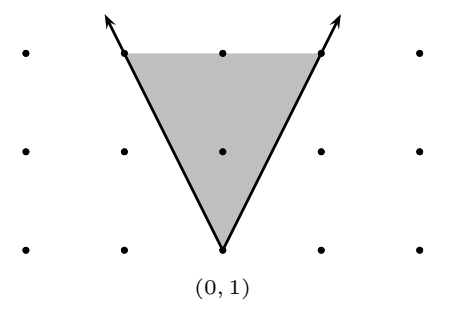

(b) $\Delta_{\infty}$

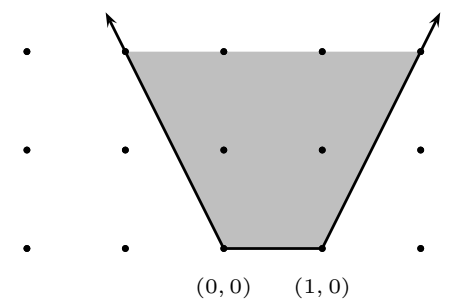

(d) $\Delta_{0}^{1}$

Figure 1. Smoothing $\mathbb{P}(1,1,2)$ to $\mathbb{P}^{1} \times \mathbb{P}^{1}$.

polynomial $f$. Then as previously noted, $f$ can be written as

$$
f=\sum_{i=k}^{l} f_{i} z_{1}^{i}
$$

with $f_{i}$ Laurent polynomials in $z_{2}, \ldots, z_{n}$ such that, for $i>0, f_{i} / g^{i}$ is a Laurent polynomial. Since we are assuming that $\Delta(f)$ contains the origin in its interior, we must have $k<0<l$.

The coordinates $z_{1}, \ldots, z_{n}$ correspond to a basis $e_{1}, \ldots, e_{n}$ of $\mathbb{Z}^{n}$. Let $N=\mathbb{Z} \oplus \mathbb{Z}^{n}$ with basis $e_{0}, \ldots, e_{n}$, and let $\sigma=\operatorname{cone}\left\{\Delta(f)+e_{0}\right\}$. Then $\mathbb{T} \mathbb{V}(\sigma)$ is the affine cone over $\mathbb{T} \mathbb{V}(\Delta(f))$, with grading given by $e_{0}$.

Now, take $u=e_{1}^{*}$, and let $s: N \rightarrow N^{\prime}$ be the cosection coming from the induced splitting of $N$. Let $\Delta_{0}$ and $\Delta_{\infty}$ be as in Section 2 and let $\tau=s((\operatorname{ker} u) \cap \sigma)$ be their common tailcone. Then $\Delta_{0}$ is the Minkowski sum of $\tau$ with the polytope

$$
\operatorname{conv}\left\{\frac{\Delta\left(f_{i}\right)}{i}+\frac{e_{0}}{i}\right\}_{i=1}^{l} .
$$

Likewise, $\Delta_{\infty}$ is the Minkowski sum of $\tau$ with the polytope

$$
\operatorname{conv}\left\{\frac{\Delta\left(f_{i}\right)}{-i}+\frac{e_{0}}{-i}\right\}_{i=k}^{-1}
$$

Taking

$$
\Delta_{0}^{0}:=\tau+\operatorname{conv}\left\{\frac{\Delta\left(f_{i} / g^{i}\right)}{i}+\frac{e_{0}}{i}\right\}_{i=1}^{l}, \quad \Delta_{0}^{1}:=\tau+\Delta(g)
$$

gives an admissible pair such that $\Delta_{0}^{0}+\Delta_{0}^{1}=\Delta_{0}$, and $\Delta_{0}^{1}, \Delta_{\infty}$ is admissible as well. Indeed, admissibility follows from the fact that $\Delta_{0}^{1}$ is a lattice polyhedron, and the identity $\Delta_{0}^{0}+\Delta_{0}^{1}=\Delta_{0}$ follows from the distributive law for convex hulls and Minkowski sums. 
We may thus use Theorem 2.1 to get a flat family $\pi: \mathcal{X} \rightarrow \mathbb{P}^{1}$ with special fibers $\mathcal{X}_{0}=\mathbb{T} \mathbb{V}(\sigma)$ and $\mathcal{X}_{\infty}$. The cone $\sigma_{\infty}$ describing $\mathcal{X}_{\infty}$ is the cone generated by $\tau$ and by elements of

$$
\Delta\left(f_{i} / g^{i}\right)+e_{0}+i e_{1}
$$

for $k \leq i \leq l, i \neq 0$.

Now, note that

$$
\phi(f)=\sum_{i=k}^{l}\left(f_{i} / g^{i}\right) z_{1}^{i} .
$$

Let $\sigma^{\prime}=\operatorname{cone}\left\{\Delta(\phi(f))+e_{0}\right\}$. In a moment, we will show that $s\left((\operatorname{ker} u) \cap \sigma^{\prime}\right)=\tau$. It then follows from the description of $\sigma_{\infty}$ that $\sigma_{\infty}=\sigma^{\prime}$. Applying Proj to the family $\pi$ thus gives us a flat projective family with special fibers $\mathbb{T} \mathbb{V}(\Delta(f))$ and $\mathbb{T} \mathbb{V}(\Delta(\phi(f)))$, proving Theorem 1.3.

It remains to be shown that $s\left((\operatorname{ker} u) \cap \sigma^{\prime}\right)=\tau$. We will only show the inclusion $\tau \subset$ $s\left((\right.$ ker $\left.u) \cap \sigma^{\prime}\right)$; the inclusion $s\left((\operatorname{ker} u) \cap \sigma^{\prime}\right) \subset \tau$ follows from an almost identical argument. So let us consider some $v \in \tau$. For $k \leq i \leq l$ we can find $\lambda_{i} \in \mathbb{Q}_{\geq 0}$ and $v_{i} \in \Delta\left(f_{i}\right)$ satisfying $\sum i \lambda_{i}=0$ such that

$$
v=\sum_{i=k}^{l} \lambda_{i} v_{i} .
$$

For $i>0$, let $v_{i}^{\prime} \in \Delta\left(f_{i} / g^{i}\right), w_{i} \in \Delta\left(g^{i}\right)$ be such that $v_{i}=v_{i}^{\prime}+w_{i}$. Set

$$
w=\frac{1}{\sum_{i=1}^{l} i \lambda_{i}} \sum_{i=1}^{l} \lambda_{i} w_{i}
$$

and note that $w \in \Delta(g)$. Indeed, for each $i>0, \frac{w_{i}}{i} \in \Delta(g)$, and $w$ is in their convex hull. Thus

$$
v=\sum_{i=1}^{l} \lambda_{i} v_{i}^{\prime}+\sum_{i=k}^{0} \lambda_{i}\left(v_{i}-i w\right)
$$

which clearly lies in $s\left((\operatorname{ker} u) \cap \sigma^{\prime}\right)$. This completes the proof of Theorem 1.3.

Example 3.1. Consider the Laurent polynomial $f=x^{-1} y+2 y+x y+y^{-1}$. The mutation $x \mapsto x, y \mapsto y /(1+x)$ sends $f$ to $f^{\prime}=x^{-1} y+y+y^{-1}+x y^{-1}$. We have $\mathbb{T} \mathbb{V}(\Delta(f))=\mathbb{P}(1,1,2)$ and $\mathbb{T} \mathbb{V}\left(\Delta\left(f^{\prime}\right)\right)=\mathbb{P}^{1} \times \mathbb{P}^{1}$, and corresponding family of Theorem 1.3 is exactly that discussed in Example 2.3.

\section{Remarks on mutations and surfaces}

We now discuss the connection between Theorem 1.3 and the mutations of [4]. Let $N$ be a rank two lattice with dual $M$, and consider some primitive element $u \in M \backslash\{0\}$. Let $e_{1}, e_{2}$ be a basis of $N$ such that $u=e_{2}^{*}$, and let $x, y$ be the corresponding monomials in $\mathbb{C}[N]$, i.e. $x=\chi^{e_{1}}$ and $y=\chi^{e_{2}}$. Galkin and Usnich consider the birational transformation

$$
\phi_{u}: \mathbb{C}(N) \rightarrow \mathbb{C}(N), \quad x \mapsto x, \quad y \mapsto \frac{y}{1+x} .
$$

Remark 4.1. Different choices of basis will arise in transformations differing by an element $\psi \in \operatorname{Aut}(\mathbb{C}(N))$ of the form $\psi\left(\chi^{u}\right)=\chi^{A(u)}$, where $A$ is an automorphism of $N$. Such a map $\psi$ always maps any Laurent polynomial $f$ to a Laurent polynomial, and $\Delta(f)$ and $\Delta(\psi(f))$ are lattice isomorphic. Thus, we will henceforth ignore the role that the choice of basis plays. 


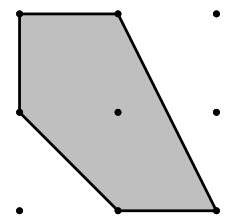

(a) $\Delta\left(f^{\prime}\right)$

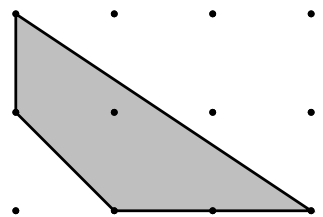

(b) $\Delta\left(\phi\left(f^{\prime}\right)\right)$

Figure 2. A mutation of $f^{\prime}$.

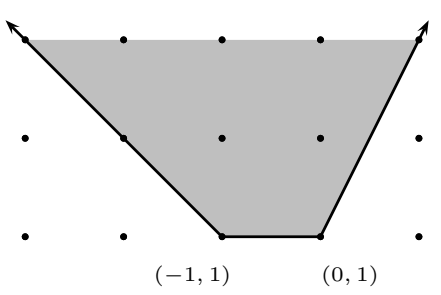

(a) $\Delta_{0}$

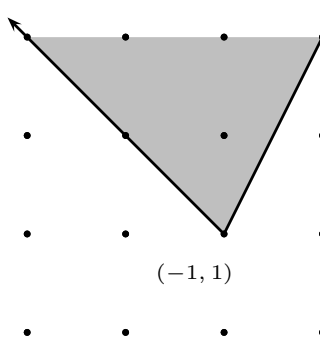

(c) $\Delta_{0}^{0}$

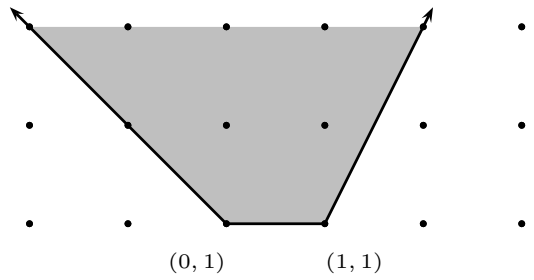

(b) $\Delta_{\infty}$

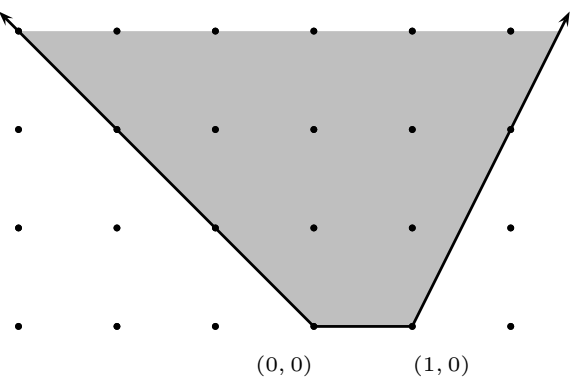

(d) $\Delta_{0}^{1}$

Figure 3. A family corresponding to a mutation.

Now, let $\Delta$ be any lattice polytope in $N_{\mathbb{Q}}$ containing the origin whose vertices are all primitive lattice vectors. Such polytopes describe all toric del Pezzo surfaces via $\mathbb{T} \mathbb{V}(\Delta)$. For any facet $\tau \prec \Delta$, let $u_{\tau}$ be the primitive element of $M$ whose maximum value on $\Delta$ is achieved exactly on $\tau$. Note that $u(\tau)$ measures the lattice height of $\tau$. Define $\phi_{\tau}$ to be the birational transformation $\phi_{u_{\tau}}$ from above.

In [4, Section 3] there is a list of ten Laurent polynomials whose constant terms series correspond to the Gromov-Witten theory of the ten different families of smooth del Pezzo surfaces. For each polynomial $f$ in the list, $\mathbb{T V}(\Delta(f))$ is a singular toric del Pezzo surface admitting a $\mathbb{Q}$ Gorenstein smoothing. Furthermore, $\phi_{\tau}$ is a mutation for each facet $\tau$, and this property holds for the new Laurent polynomial $\phi_{\tau}(f)$ and subsequent mutations thereof as well, see [4, Theorem 16]. For each polynomial $f$, we can thus define an infinite graph $\Gamma_{f}$ whose vertices are Laurent polynomials attained from $f$ via such mutations, and whose edges correspond to mutations.

To any edge of one of the above graphs $\Gamma_{f}$, Theorem 1.3 says that we can associate a deformation between the toric varieties corresponding to that edge's vertices.

Example 4.2. Consider the Laurent polynomial $f=x+y+x^{-1} y^{-1}+x^{-1}+y^{-1}$ from the list of [4] corresponding to the del Pezzo surface of degree seven. This is lattice equivalent to the 
polynomial $f^{\prime}=x^{-1}+x^{-1} y+y+y^{-1}+x y^{-1}$. Mutating this polynomial (with respect to this choice of $x, y)$ gives $\phi\left(f^{\prime}\right)=x^{-1}+x^{-1} y+y^{-1}+x y^{-1}+x^{2} y^{-1}$. In Fig. 2, we picture the Newton polytopes of $f^{\prime}$ and $\phi\left(f^{\prime}\right)$. In Fig. 3 we picture the polytopes $\Delta_{0}, \Delta_{\infty}, \Delta_{0}^{0}$, and $\Delta_{0}^{1}$ as in the proof of Theorem 1.3.

\section{References}

[1] Altmann K., Hausen J., Polyhedral divisors and algebraic torus actions, Math. Ann. 334 (2006), 557-607, math.AG/0306285.

[2] Auroux D., Mirror symmetry and T-duality in the complement of an anticanonical divisor, J. Gökova Geom. Topol. GGT 1 (2007), 51-91, arXiv:0706.3207.

[3] Fulton W., Introduction to toric varieties, Annals of Mathematics Studies, Vol. 131, Princeton University Press, Princeton, NJ, 1993.

[4] Galkin S., Usnich A., Mutations of potentials, Preprint IPMU 10-0100.

[5] Ilten N.O., Vollmert R., Deformations of rational T-varieties, J. Algebraic Geom. 21 (2012), 531-562, arXiv:0903.1393.

[6] Mavlyutov A., Deformations of toric varieties via Minkowski sum decompositions of polyhedral complexes, arXiv:0902.0967. 